CORRIGENDUM

doi:10.1038/nature 18281

\title{
Corrigendum: Mycocerosic acid synthase exemplifies the architecture of reducing polyketide synthases
}

Dominik A. Herbst, Roman P. Jakob, Franziska Zähringer

\& Timm Maier

Nature 531, 533-537 (2016); doi:10.1038/nature16993

In this Letter, we studied the three-dimensional structure of a protein from Mycobacterium smegmatis assigned as mycocerosic acid synthase (MAS) in sequence databases as A0R1E8 in Uniprot (http://www. uniprot.org/uniprot/A0R1E8) and YP_888986.1 in NCBI (https://www. ncbi.nlm.nih.gov/protein/118473069). In conclusion, we provided a template structure of MAS-like polyketide synthases (PKSs) and a first example of reducing PKS architecture. However, we now note that Etienne et al. ${ }^{1}$ provided a biochemical characterization of a deletion strain of the corresponding gene MSMEG_4727 (https://www.ncbi. nlm.nih.gov/gene/4534621), which indicated a physiological role of the protein in the production of 2,4-dimethyl-2-eicosenoic acid, a lipid component of lipooligosaccharides, rather than mycocerosic acids, via a reaction closely related to those of MAS. Until comprehensive characterization at the protein level is available, the protein we studied should therefore be referred to as a 'mycocerosic-acid synthase like-PKS' or 'MAS-like PKS'; the database records will be updated accordingly. We thank the authors of ref. 1 for drawing our attention to this publication. The main scientific conclusions of our manuscript remain unchanged.

1. Etienne, G. et al. Identification of the polyketide synthase involved in the biosynthesis of the surface-exposed lipooligosaccharides in mycobacteria. J. Bacteriol. 191, 2613-2621 (2009). 